\title{
O papel do lactato na regulação neuroendócrina do eixo somatotrófico.
}

Tese apresentada ao Programa de Pósgraduação em Fisiologia Humana do Instituto de Ciências Biomédicas da Universidade de São Paulo, para obtenção do Título de Doutor em Ciências.

Área de concentração: Fisiologia Humana Orientadora: $\operatorname{Prof}^{\mathrm{a}} \operatorname{Dr}^{\mathrm{a}}$ Maria Tereza Nunes

Versão corrigida. A versão original encontrase disponível na Secretaria de Pós-graduação que aloja o Programa de Pós-Graduação.

SÃO PAULO

2018 


\section{RESUMO}

Lactato é o produto da redução do piruvato, reação fundamental para controle intracelular do pH e o estado redox celular. Na corrente sanguínea, ele se difunde aos tecidos que expressam MCTs e é consumido como um importante substrato energético. Nos estudos mostraram que o lactato promove elevação da síntese/secreção do GH em ratos. Neste estudo, pretendemos identificar se esse metabólito poderia estimular diretamente as células hipotalâmicas e/ou hipofisárias relacionadas com eixo somatotrófico, bem como seus potenciais mecanismos envolvidos nesse efeito. Nossos resultados mostram que o lactato aumenta a atividade de neurônios em diferentes regiões hipotalâmicas, elevando a expressão gênica de Ghrh e Somatostatina (Sst). Essas alterações estariam regulando efeitos pós-transcricionais sobre o mRNA de $G h$, o que pode refletir na maior estabilidade e no conteúdo do seu transcrito. Os efeitos do lactato também foram estudados em animais knockout para receptor de GHRH, mostrando a importância de um tônus estimulatório do GHRH no aumento do conteúdo sérico do GH. Em experimentos com camundongos $\mathrm{KO} \mathrm{MCT}_{1+/-}$ demonstramos que o lactato é fundamental para a ativação do eixo somatotrófico em resposta ao exercício. Ainda, identificamos a expressão do receptor de lactato $\left(\right.$ Gpcr $\left._{81}\right)$ e de 3 diferentes isoformas de transportadores de lactato $\left(M c t_{1,3,4}\right)$ de células $\mathrm{GH} 3$, nas quais também detectamos elevação do conteúdo intra e extracelular de GH em resposta ao tratamento com lactato. Este último efeito indica o seu estímulo sobre a secreção de $\mathrm{GH}$, que se mostrou dependente do influxo de $\mathrm{Ca}^{2+}$ extracelular. Frente à inibição farmacológica dos MCTs, o efeito do lactato sobre o aumento do mRNA de $G h$ não é mais reproduzido. Em suma, utilizando diferentes abordagens experimentais, demonstramos que o lactato atua diretamente nos somatotrofos, via transportadores de membrana, promovendo aumento da síntese e secreção de GH, bem como no hipotálamo, aumentando a expressão de GHRH e SST. Conclui-se, portanto, que o lactato promove aumento da atividade do eixo somatotrófico atuando em diferentes níveis do mesmo, o que pode contribuir com a elevada concentração do GH em resposta ao exercício físico e o coloca como um potente sinalizador central de significativas alterações metabólicas corpóreas.

Palavras-chave: Hipotálamo, Metabolismo, Hormônio do Crescimento, Eixo somatotrófico, Lactato. 


\section{ABSTRACT}

Lactate is the product of pyruvate reduction, a fundamental reaction for intracellular $\mathrm{pH}$ control and the redox state of the cell. In the bloodstream, it diffuses into tissues expressing MCTs and is consumed as an important energy substrate. In my master's degree, we observed that lactate promotes elevation of GH synthesis/ secretion in rats. In this study, we intend to identify whether this metabolite could stimulate directly the hypothalamic and/or pituitary cells related to the somatotropic axis, as well as the potential mechanisms involved in this regulation. Our results show that lactate increases the activity of neurons in different hypothalamic regions, regulating the gene and protein expression of Ghrh and Somatostatin (Sst). Those events could be promoting post-transcriptional regulation of the Gh mRNA, which may reflect the increment of its transcript stability and content. In experiments with Lit/Lit mice, we observed the importance of stimulating GHRH tone for the stimulation of GH expression. In experiments with $\mathrm{MCT}_{1}{ }^{+-} \mathrm{KO}$ mice we identified that lactate is a key part of exercise to activate the somatotrofic axis. Furthermore, we characterized that GH3 cells show lactate receptor mRNA expression $\left(G_{p c r} r_{81}\right)$ and 3 different isoforms of lactate transporters $\left(M c t_{1,3,4}\right)$, in which we detect the intra and extra cellular GH content increased as a result of the lactate treatment. This last effect indicates a stimulus on the GH secretion, which is dependent of the extracellular $\mathrm{Ca}^{2+}$ influx. Facing pharmacological inhibition of MCTs, the effect of lactate on the Gh mRNA increment is no longer reproduced. Thus, applying different experimental approaches, our work shows direct lactate actions, via membrane transporters, promoting increased GH synthesis and secretion, as well as hypothalamic expression of GHRH and SST. We concluded, therefore, that lactate promotes an increase in the activity of the somatotrofic axis acting at different levels of the same, which can contribute to the high concentration of GH in response to physical exercise and places it as a potent central signaling factor of the significant body metabolic alterations.

Key words: Hypothalamus, Metabolism, Growth Hormone, Somatotrofic axis, Lactate. 


\section{INTRODUÇÃO}

O lactato é um ânion gerado a partir da redução do piruvato proveniente do processo de metabolização anaeróbia da glicose, por meio de uma reação catalisada pela enzima lactato desidrogenase (LDH). Esta utiliza os íons $\mathrm{H}^{+}$e $\mathrm{NADH}$ acumulados no citossol, gerando $\mathrm{NAD}^{+}$.

Figura 1 - Reação piruvato/lactato

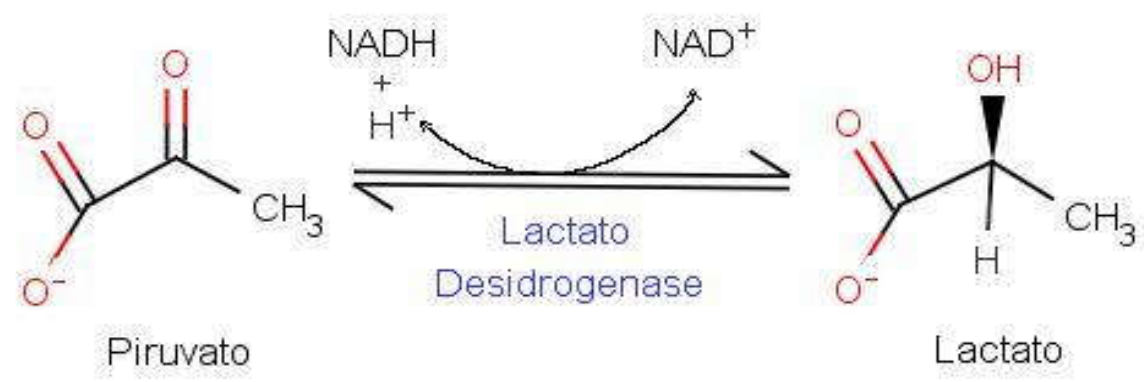

Reação redox bidirecional de piruvato/lactato catalisada pela enzima lactato desidrogenase.

Quando a oferta de oxigênio está adequada às necessidades do tecido, o piruvato resultante da glicólise é preferencialmente oxidado dentro da mitocôndria a $\mathrm{CO}_{2}$ e $\mathrm{H}_{2} \mathrm{O}$ pelo ciclo de Krebs. Essa alimentação da cadeia respiratória na membrana interna da mitocôndria, juntamente com a utilização de coenzimas, permite a fosforilação do ADP a ATP, caracterizando um dos maiores processos de fonte de ATP intracelular.

Durante uma atividade física de alta intensidade e/ou de grande volume de esforço, a concentração de lactato na corrente sanguínea alcança valores elevados, o que depende do estado de treinamento físico do atleta. Neste contexto, análises da concentração plasmática de lactato de indivíduos sedentários que foram submetidos à corrida de longa distância mostraram uma elevação na ordem de $13,4 \mathrm{mM}$ e o mesmo protocolo de esforço físico aplicado a indivíduos treinados a corridas de longa distância resultou em um pico de lactato ao redor de 9,7 mM (THOMAS et al., 2005).

Esse aumento advém de condições específicas pelas quais as células, principalmente as musculares, são submetidas. Este fenômeno resulta no aumento do consumo energético proveniente da contração muscular, que culmina no alcance do limiar máximo do oxigênio ofertado pelos pulmões e que não supre a demanda exigida pela mitocôndria. Assim, a 
produção de ATP pela fosforilação oxidativa sofre saturação, sendo limitada pela atividade da enzima piruvato desidrogenase mitocondrial (GRANCHI et al., 2010).

Assim, com a diminuição da oxidação do piruvato na mitocôndria e com a contínua utilização da glicose devido à manutenção de elevada necessidade energética, o resultado é o acúmulo de piruvato e de íons $\mathrm{H}^{+}$no citossol. Esse resíduo da glicólise servirá de substrato para a enzima lactato desidrogenase, que favorecerá a reação de síntese do lactato intracelular.

O acúmulo do lactato citossólico culmina no seu efluxo para a corrente sanguínea, via transportadores transmembrânicos de monocarboxilato $\left(\mathrm{MCT}_{1} \mathrm{a}_{4}\right)$, por gradiente de concentração (ZAJAC; WAŚKIEWICZ; PILIS, 2001). Esse processo requer o cotransporte de outro íon $\mathrm{H}^{+}$na proporção $1: 1$, sendo este o mecanismo que mais contribui para a acidose metabólica do organismo, já que soma $50 \%$ dos íons $\mathrm{H}^{+}$circulantes durante o esforço físico, o que é essencial para a manutenção do pH intracelular (PILEGAARD et al., 1999).

A captação de lactato pelas células hepáticas é mediada por 3 principais isoformas de MCTs (1, 2 e 4). O MCT 1 , que exibe $\mathrm{Km}$ de 3,5 mM para lactato, é o mais importante dos MCTs e apresenta expressão ubíqua nos diferentes tecidos. É interessante comentar que o knockout de $\mathrm{MCT}_{1}$ em camundongos promove uma resistência à aquisição de obesidade induzida pela dieta (LENGACHER et al., 2013), o que sugere que esses transportadores estejam envolvidos no controle do balanço energético corpóreo. Os $\mathrm{MCT}_{2} \mathrm{e}_{4}$ apresentam Kms para lactato diferentes do $\mathrm{MCT}_{1}$ e, ao contrário deste, são expressos de forma tecidoespecífica. $\mathrm{O}_{\mathrm{MCT}_{2}}(\mathrm{Km}$ de $0,74 \mathrm{mM})$ é o principal transportador de lactato neuronal,

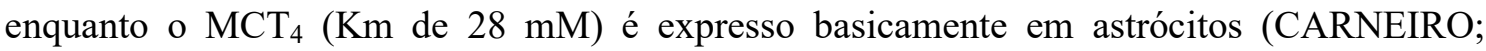
PELLERIN, 2015). Além desses transportadores, existe a expressão do $\mathrm{MCT}_{3}$, que foi exclusivamente identificado no epitélio de retina (YOON et al., 1997).

Uma vez na circulação, o lactato se difunde pela membrana das células que expressam MCTs e que não estão saturados de lactato, exercendo assim diferentes papéis fisiológicos nessas células. No fígado, ele é oxidado novamente a piruvato, proporcionando uma rica fonte de carbono para a gliconeogênese, contribuindo para a manutenção da glicemia.

O conjunto desses transportadores possibilita o fluxo de lactato entre sangue e tecidos, em resposta à variação da sua concentração. Assim, nos músculos com predominância de fibras oxidativas há maior quantidade de transportadores de lactato, o que permite sua captação e oxidação à piruvato, substrato fundamental para a produção de energia pelo ciclo de Krebs (LING et al., 2012; MCCULLAGH et al. 1996). Do mesmo modo, YANASE e colaboradores (2008) identificaram diversas subunidades de MCTs dependentes de sódio na membrana basolateral e na membrana apical do túbulo renal de camundongos, dado indicativo 
de que o lactato também pode ser excretado na urina, bem como utilizado como fonte de energia.

Sabe-se ainda que o lactato atravessa a barreira hematoencefálica e pode atingir células nervosas, de forma a atuar como substrato energético e/ou um importante sinalizador central (SMITH et al., 2003). Esse ânion pode ser consumido pelas células nervosas durante a atividade física, chegando a poupar cerca de $1 / 3$ de todos os outros substratos utilizados pelo cérebro, como glicose e corpos cetônicos (RASMUSSEN, WYSS e LUNDBY, 2011; IDE e SECHER, 2000; VAN HALL et al., 2009; OVERGAARD et al., 2012).

Por meio da metabolização da glicose sérica pelas células da glia, que constituem cerca de $90 \%$ das células neurais, o lactato gerado é rapidamente transferido para os neurônios adjacentes por via de Lançadeiras de Lactato dos Astrócitos para os Neurônios (ANLS) (MAGISTRETTI; PELLERIN, 1999).

Estudo de Parsons e Hirasawa (2010) mostrou que o bloqueio dos MCTs com ácido alfa-ciano-4-hidroxicinâmico, em neurônios orexígenos depletados de glicose e com elevadas concentrações de lactato de sódio no meio de cultura, provoca o desaparecimento total da sua freqüência de disparos. Esta retorna ao basal no momento em que há o desbloqueio desses transportadores, sugerindo a participação do lactato no desencadeamento desses potenciais de ação, o colocando como um importante sinalizador metabólico.

As mais novas descobertas científicas colocam-no também como um importante regulador da atividade respiratória. Com o rápido aumento da sua concentração sanguínea em estado de hipóxia, o lactato ativa receptores Olf78 acoplados à proteína $G$ de células quimiossensíveis do corpo carotídeo, despolarizando-as, o que contribui com a hiperventilação gerada nesse estado (CHANG et al., 2015).

Considerando a multiplicidade de eventos desencadeados por ele e sabendo-se que sua concentração sanguínea se eleva durante o exercício, situação em que ocorre um aumento da secreção de GH, por mecanismos já identificados (DELITALA et al., 1987; SHIBASAKI et al., 1985; KOJIMA et al., 1999), desenvolvemos um estudo que mostrou que a administração de lactato em ratos em repouso promoveu uma elevação da secreção do GH (SALGUEIRO et al., 2014), o que sugere sua contribuição para o aumento do GH no exercício, hormônio expresso pelas células somatotróficas, e regulado por diversos fatores, entre eles o estado metabólico (SPIESS; RIVIER; VALE, 1983; TANNENBAUM; BOWERS, 2001).

Nossos dados mostraram que ratos tratados com uma única injeção de lactato nas concentrações de 15 e $150 \mu$ mols, durante a fase escura (escotofase) do período de $24 \mathrm{~h}$, apresentaram aumento da expressão gênica de Gh e Igf-1 (SALGUEIRO et al., 2014). Esses 
dados dão destaque à importância do lactato na ativação do eixo somatotrófico e sugerem sua ação sobre a atividade das células somatotróficas hipofisárias e/ou de neurônios hipotalâmicos que controlam a secreção de $\mathrm{GH}$, hormônio que participa da manutenção da homeostase energética durante o exercício (GODFREY; MADGWICK; WHYTE, 2003).

Nosso estudo vai ao encontro de pesquisas em humanos que mostraram que durante o treinamento exaustivo em esteira (KRAEMER e RATAMESS, 2005; ROJAS VEGA et al., 2006) ou com a infusão de lactato (SCHIFFER et al., 2011) ocorre aumento da produção de brain derived neurotrophic factor (BDNF), fatores de crescimento e da liberação de hormônios como prolactina, que poderiam estar associados aos efeitos benéficos da atividade física (DE MEIRLEIR et al., 1985; ROJAS VEJA, STRÜDER e HOLLMANN, 2003; ROJAS VEGA et al., 2006).

Além de agentes metabólicos, os principais reguladores da atividade dos somatotrofos são os hormônios produzidos por neurônios hipotalâmicos, entre eles o Hormônio Liberador de GH (GHRH) e a Somatostatina (SST). Os corpos celulares dos neurônios GHRH estão localizados principalmente nos núcleos arqueado e ventromedial do hipotálamo, enquanto que os da SST na região periventricular (FODOR et al., 1994).

O GHRH foi caracterizado e sequenciado pelos grupos Guillemin e de Rivier (1982), e qualificado como um importante estimulador da secreção e síntese de GH, por ativar receptores transmembrânicos acoplados à proteína G estimulatória (Gs) (ROOT; ROOT, 2002). O estudo de Kamegai et al. (1998), em ratos espontaneamente anões (SDR), demonstrou que a deficiência de GH promove aumento da expressão do GHRH e de seus receptores GHRH-r, bem como redução da síntese de neuropeptídio Y (NPY) e SST, e que sua reposição exógena promove efeito inverso, demonstrando a importância do GH no controle da atividade de seu eixo.

A SST também é reconhecida por células somatotróficas regulando negativamente a produção de GH. A ação da SST ocorre por meio da ativação do receptor de SST, que é acoplado a proteína $\mathrm{G}$ inibitória (Gi), e resulta na diminuição da secreção e síntese de $\mathrm{GH}$ (ROOT; ROOT, 2002). Lesões no núcleo periventricular do hipotálamo, que possibilitaram uma redução de cerca de $80 \%$ do seu conteúdo na eminência mediana e 30\% no próprio hipotálamo, foram seguidos de aumento na concentração sanguínea de GH (CRITCHLOW et al., 1981).

Nesse sentido, Wideman et al. (1999) mostraram que 30 min de exercício aeróbio de alta intensidade é eficiente para elevar a concentração sérica de GH a seu máximo em homens e mulheres adultos, atribuindo para tal resposta a combinação do aumento na secreção de 
GHRH com a supressão da secreção de SST. Considerando que durante um exercício intenso essa região é banhada por altas concentrações de lactato, é possível que esse metabólito participe da secreção de GHRH.

O hipotálamo endócrino é uma importante região do sistema nervoso central que recebe inúmeras projeções de outras partes do cérebro e sinais provindos da circulação sistêmica. A somatória desses estímulos resulta no controle endócrino da hipófise. Há evidências de que neurônios hipotalâmicos que expressam abundantemente o MCT1 apresentam elevação das concentrações de ATP e NADH em resposta ao lactato, e não ao piruvato (AINSCOW et al., 2002). Assim, o aumento da concentração do lactato circulante poderia alterar o fluxo deste metabólito para as células e desencadear eventos nas mesmas, contribuindo para o controle da liberação de neurohormônios.

O conjunto dos dados apresentados coloca o lactato como um fator adicional que contribuiria para o aumento do GH em resposta ao exercício ou ao estado metabólico do indivíduo, atuando como um sinalizador da perturbação da homeostase energética (SALGUEIRO et al., 2014). No presente trabalho, exploramos a importância deste metabólito para a ativação do eixo somatotrófico, buscando identificar os mecanismos pelos quais seus efeitos são desencadeados. 


\section{CONCLUSÃO}

Podemos concluir que o lactato pode ser considerado um potente estimulador do eixo somatotrófico. Em ações sobre o sistema nervoso central, ele aumenta o conteúdo de transcritos de neurohormônios hipotalâmicos envolvidos com a síntese e secreção de GH, bem como aumenta a atividade de outros neurônios hipotalâmicos. O mesmo foi capaz de aumentar a estabilidade e o conteúdo do mRNA de Gh, e o GH sérico, contudo seus efeitos são intrinsecamente dependentes da expressão de transportadores nos tecidos alvos, conforme demonstrado nos estudos in vivo.

Ainda, nosso trabalho caracterizou células somatolactotróficas quanto à expressão de transportadores e receptores relacionados à ação do lactato sobre as mesmas, possibilitando compreender a resposta de aumento no conteúdo e secreção de GH deve-se a mecanismos dependentes de seu influxo, bem como de um consecutivo influxo de cálcio, como observado no estudo in vitro.

Assim, nossos achados corroboram os dados que apontam o lactato como um elemento importante para a atividade hipotalâmica em geral e, principalmente, que ele é um agente estimulador da atividade do eixo somatotrófico nos diferentes tecidos que o constituem, conforme fora sugerido na hipótese inicial do mestrado. Esses dados, além de acrescentarem novas informações a este campo do conhecimento, levanta a possibilidade de uma perspectiva terapêutica para o lactato, rompendo o paradigma de que ele seja apenas um produto de descarte no metabolismo da glicose e causador de acidose metabólica. 


\section{REFERÊNCIAS ${ }^{*}$}

AINSCOW, E. K. et al. Dynamic imaging of free cytosolic ATP concentration during fuel sensing by rat hypothalamic neurones: evidence for ATP-independent control of ATPsensitive $\mathrm{K}(+)$ channels. The Journal of Physiology, v. 544, n. Pt 2, p. 429-445, 15 out. 2002.

ALJADA, A. et al. Insulin inhibits the pro-inflammatory transcription factor early growth response gene-1 (Egr)-1 expression in mononuclear cells (MNC) and reduces plasma tissue factor (TF) and plasminogen activator inhibitor-1 (PAI-1) concentrations. The Journal of Clinical Endocrinology and Metabolism, v. 87, n. 3, p. 1419-1422, mar. 2002.

BERNSTEIN, P.; ROSS, J. Poly(A), poly(A) binding protein and the regulation of mRNA stability. Trends in Biochemical Sciences, v. 14, n. 9, p. 373-377, set. 1989.

BLOCH, B. et al. Immunohistochemical detection of growth hormone-releasing factor in brain. Nature, v. 301, n. 5901, p. 607-608, 17 fev. 1983.

BLOUET, C.; SCHWARTZ, G. J. Hypothalamic nutrient sensing in the control of energy homeostasis. Behavioural Brain Research, v. 209, n. 1, p. 1-12, 1 maio 2010.

CARNEIRO, L. et al. Evidence for hypothalamic ketone body sensing: impact on food intake and peripheral metabolic responses in mice. American Journal of Physiology. Endocrinology and Metabolism, v. 310, n. 2, p. E103-115, 15 jan. 2016.

CARNEIRO, L.; PELLERIN, L. Monocarboxylate transporters: new players in body weight regulation. Obesity Reviews: An Official Journal of the International Association for the Study of Obesity, v. 16 Suppl 1, p. 55-66, fev. 2015.

CHOMCZYNSKI, P.; SACCHI, N. The single-step method of RNA isolation by acid guanidinium thiocyanate-phenol-chloroform extraction: twenty-something years on. Nature protocols, v. 1, n. 2, p. 581-585, 2006.

CORRÊA-DE-SANTANA, E. et al. Modulation of growth hormone and prolactin secretion in Trypanosoma cruzi-infected mammosomatotrophic cells. Neuroimmunomodulation, v. 16, n. 3, p. 208-212, 2009.

CORTES-CAMPOS, C. et al. MCT2 expression and lactate influx in anorexigenic and orexigenic neurons of the arcuate nucleus. PloS one, v. 8, n. 4, p. e62532, 2013.

DE MEIRLEIR, K. L. et al. Exercise-induced prolactin release is related to anaerobiosis. The Journal of clinical endocrinology and metabolism, v. 60, n. 6, p. 1250-1252, jun. 1985.

DELITALA, G. et al. Dopaminergic and cholinergic influences on the growth hormone response to growth hormone-releasing hormone in man. Neuroendocrinology, v. 45, n. 3, p. 243-247, mar. 1987.

DEVESA, J.; LIMA, L.; TRESGUERRES, J. A. F. Neuroendocrine control of growth hormone secretion in humans. Trends in Endocrinology \& Metabolism, v. 3, n. 5, p. 175 183, jul. 1992.

* De acordo com: ASSOCIAÇÃO BRASILEIRA DE NORMAS TÉCNICAS. NBR 6023: informação e documentação: referências: elaboração. Rio de Janeiro, 2002. 
FODOR, M. et al. Growth hormone-releasing hormone, somatostatin, galanin and betaendorphin afferents to the hypothalamic periventricular nucleus. Journal of chemical neuroanatomy, v. 8, n. 1, p. 61-73, nov. 1994.

FROHMAN, L. A.; NERNARDIS, L. L.; KANT, K. J. Hypothalamic stimulation of growth hormone secretion. Science (New York, N.Y.), v. 162, n. 3853, p. 580-582, 1 nov. 1968.

GODFREY, R. J.; MADGWICK, Z.; WHYTE, G. P. The exercise-induced growth hormone response in athletes. Sports medicine (Auckland, N.Z.), v. 33, n. 8, p. 599-613, 2003.

GRANCHI, C. et al. Inhibitors of Lactate Dehydrogenase Isoforms and their Therapeutic Potentials. Current Medicinal Chemistry, v. 17, n. 7, p. 672-697, 1 mar. 2010.

GUAN, X. M. et al. Distribution of mRNA encoding the growth hormone secretagogue receptor in brain and peripheral tissues. Brain Research. Molecular Brain Research, v. 48, n. 1, p. 23-29, ago. 1997.

GUILLEMIN, R. et al. Growth hormone-releasing factor from a human pancreatic tumor that caused acromegaly. Science (New York, N.Y.), v. 218, n. 4572, p. 585-587, 5 nov. 1982.

HOQUE, R. et al. Lactate Reduces Liver and Pancreatic Injury in Toll-Like Receptor- and Inflammasome-Mediated Inflammation via GPR81-Mediated Suppression of Innate Immunity. Gastroenterology, v. 146, n. 7, p. 1763-1774, jun. 2014.

IDE, K.; SECHER, N. H. Cerebral blood flow and metabolism during exercise. Progress in Neurobiology, v. 61, n. 4, p. 397-414, jul. 2000.

ITOH, K. et al. Application of coupled oxidation reaction to electron microscopic demonstration of horseradish peroxidase: cobalt-glucose oxidase method. Brain research, $\mathrm{v}$. 175, n. 2, p. 341-346, 19 out. 1979.

KAMEGAI, J. et al. Hypothalamic/pituitary-axis of the spontaneous dwarf rat: autofeedback regulation of growth hormone $(\mathrm{GH})$ includes suppression of $\mathrm{GH}$ releasing-hormone receptor messenger ribonucleic acid. Endocrinology, v. 139, n. 8, p. 3554-3560, ago. 1998.

KOJIMA, M. et al. Ghrelin is a growth-hormone-releasing acylated peptide from stomach. Nature, v. 402, n. 6762, p. 656-660, 9 dez. 1999.

KRAEMER, W. J.; RATAMESS, N. A. Hormonal responses and adaptations to resistance exercise and training. Sports medicine (Auckland, N.Z.), v. 35, n. 4, p. 339-361, 2005.

LENGACHER, S. et al. Resistance to diet-induced obesity and associated metabolic perturbations in haploinsufficient monocarboxylate transporter 1 mice. PloS One, v. 8, n. 12, p. e82505, 2013.

LING, B. et al. D-Lactate altered mitochondrial energy production in rat brain and heart but not liver. Nutrition \& metabolism, v. 9, n. 1, p. 6, 2012.

LIPOSITS, Z. et al. Synaptic communication between somatostatinergic axons and growth hormone-releasing factor (GRF) synthesizing neurons in the arcuate nucleus of the rat. Histochemistry, v. 89, n. 3, p. 247-252, 1988. 
MAEKAWA, F. et al. Basal and stimulated lactate fluxes in primary cultures of astrocytes are differentially controlled by distinct proteins. Journal of Neurochemistry, v. 107, n. 3, p. 789-798, nov. 2008.

MAGISTRETTI, P. J.; PELLERIN, L. Astrocytes Couple Synaptic Activity to Glucose Utilization in the Brain. News in physiological sciences: an international journal of physiology produced jointly by the International Union of Physiological Sciences and the American Physiological Society, v. 14, p. 177-182, out. 1999.

MAITER, D. et al. Different effects of intermittent and continuous growth hormone (GH) administration on serum somatomedin-C/insulin-like growth factor I and liver GH receptors in hypophysectomized rats. Endocrinology, v. 123, n. 2, p. 1053-1059, ago. 1988.

MCCULLAGH, K. J. et al. Role of the lactate transporter (MCT1) in skeletal muscles. The American journal of physiology, v. 271, n. 1 Pt 1, p. E143-150, jul. 1996.

MORRISON, B. M. et al. Deficiency in monocarboxylate transporter 1 (MCT1) in mice delays regeneration of peripheral nerves following sciatic nerve crush. Experimental Neurology, v. 263, p. 325-338, jan. 2015.

MUCCIOLI, G. et al. Specific receptors for synthetic GH secretagogues in the human brain and pituitary gland. The Journal of Endocrinology, v. 157, n. 1, p. 99-106, abr. 1998.

NELSON, R. J.; TRAINOR, B. C. Neural mechanisms of aggression. Nature Reviews. Neuroscience, v. 8, n. 7, p. 536-546, jul. 2007.

OVERGAARD, M. et al. Hypoxia and exercise provoke both lactate release and lactate oxidation by the human brain. The FASEB Journal, v. 26, n. 7, p. 3012-3020, 7 jan. 2012.

PARSONS, M. P.; HIRASAWA, M. ATP-sensitive potassium channel-mediated lactate effect on orexin neurons: implications for brain energetics during arousal. The Journal of neuroscience: the official journal of the Society for Neuroscience, v. 30, n. 24, p. 80618070,16 jun. 2010.

PILEGAARD, $\mathrm{H}$. et al. Distribution of the lactate/H+ transporter isoforms MCT1 and MCT4 in human skeletal muscle. The American journal of physiology, v. 276, n. 5 Pt 1, p. E843848, maio 1999.

RASMUSSEN, P.; WYSS, M. T.; LUNDBY, C. Cerebral glucose and lactate consumption during cerebral activation by physical activity in humans. The FASEB Journal, v. 25, n. 9, p. 2865-2873, 9 jan. 2011.

REZAEE, S.; KAHRIZI, S.; HEDAYATI, M. Hormonal responses of combining enduranceresistance exercise in healthy young men. The Journal of sports medicine and physical fitness, v. 54, n. 2, p. 244-251, abr. 2014.

RHODES, L. et al. Capromorelin: a ghrelin receptor agonist and novel therapy for stimulation of appetite in dogs. Veterinary Medicine and Science, v. 4, n. 1, p. 3-16, fev. 2018.

RIVIER, J. et al. Characterization of a growth hormone-releasing factor from a human pancreatic islet tumour. Nature, v. 300, n. 5889, p. 276-278, 18 nov. 1982. 
RODRÍGUEZ-PACHECO, F. et al. Ghrelin induces growth hormone secretion via a nitric oxide/cGMP signalling pathway. Journal of Neuroendocrinology, v. 20, n. 3, p. 406-412, mar. 2008.

ROJAS VEGA, S. et al. Bicarbonate reduces serum prolactin increase induced by exercise to exhaustion. Medicine and science in sports and exercise, v. 38, n. 4, p. 675-680, abr. 2006.

ROJAS VEGA, S.; STRÜDER, H. K.; HOLLMANN, W. Plasma prolactin concentration increases after hypercapnia acidosis. Hormone and metabolic research $=$ Hormon- und Stoffwechselforschung $=$ Hormones et métabolisme, v. 35, n. 10, p. 598-601, out. 2003.

ROOT, A. W.; ROOT, M. J. Clinical pharmacology of human growth hormone and its secretagogues. Current drug targets. Immune, endocrine and metabolic disorders, v. 2, n. 1, p. $27-52$, abr. 2002.

RUTTER, G. A. et al. Pancreatic $\beta$-cell identity, glucose sensing and the control of insulin secretion. The Biochemical Journal, v. 466, n. 2, p. 203-218, 1 mar. 2015.

SALGUEIRO, R. B. et al. Lactate activates the somatotropic axis in rats. Growth hormone \& IGF research: official journal of the Growth Hormone Research Society and the International IGF Research Society, 2 out. 2014.

SANTULLI, G. et al. Calcium release channel RyR2 regulates insulin release and glucose homeostasis. The Journal of Clinical Investigation, v. 125, n. 5, p. 1968-1978, maio 2015.

SCHIFFER, T. et al. Lactate infusion at rest increases BDNF blood concentration in humans. Neuroscience letters, v. 488, n. 3, p. 234-237, 25 jan. 2011.

SHAMMAH-LAGNADO, S. ; ALHEID, G. .; HEIMER, L. Afferent connections of the interstitial nucleus of the posterior limb of the anterior commissure and adjacent amygdalostriatal transition area in the rat. Neuroscience, v. 94, n. 4, p. 1097-1123, nov. 1999.

SHIBASAKI, T. et al. Plasma GH responses to GHRH and insulin-induced hypoglycemia in man. The Journal of clinical endocrinology and metabolism, v. 60, n. 6, p. 1265-1267, jun. 1985.

SMITH, D. et al. Lactate: a preferred fuel for human brain metabolism in vivo. Journal of cerebral blood flow and metabolism: official journal of the International Society of Cerebral Blood Flow and Metabolism, v. 23, n. 6, p. 658-664, jun. 2003.

SPIESS, J.; RIVIER, J.; VALE, W. Characterization of rat hypothalamic growth hormonereleasing factor. Nature, v. 303, n. 5917, p. 532-535, 9 jun. 1983.

TANNENBAUM, G. S.; BOWERS, C. Y. Interactions of growth hormone secretagogues and growth hormone-releasing hormone/somatostatin. Endocrine, v. 14, n. 1, p. 21-27, fev. 2001.

THOMAS, C. et al. Monocarboxylate transporters, blood lactate removal after supramaximal exercise, and fatigue indexes in humans. Journal of Applied Physiology, v. 98, n. 3, p. 804809, mar. 2005. 
VAN HALL, G. et al. Blood lactate is an important energy source for the human brain. Journal of cerebral blood flow and metabolism: official journal of the International Society of Cerebral Blood Flow and Metabolism, v. 29, n. 6, p. 1121-1129, jun. 2009.

WIDEMAN, L. et al. Effects of gender on exercise-induced growth hormone release. Journal of applied physiology (Bethesda, Md.: 1985), v. 87, n. 3, p. 1154-1162, set. 1999.

WIDEMAN, L. et al. Growth hormone release during acute and chronic aerobic and resistance exercise: recent findings. Sports medicine (Auckland, N.Z.), v. 32, n. 15, p. 987 1004, 2002.

WU, S. N.; LI, H. F.; CHIANG, H. T. Characterization of ATP-sensitive potassium channels functionally expressed in pituitary GH3 cells. The Journal of Membrane Biology, v. 178, $\mathrm{n}$. 3, p. 205-214, 1 dez. 2000.

YANASE, H. et al. Cellular expression of a sodium-dependent monocarboxylate transporter (Slc5a8) and the MCT family in the mouse kidney. Histochemistry and cell biology, v. 130, n. 5, p. 957-966, nov. 2008.

YOON, H. et al. Identification of a unique monocarboxylate transporter (MCT3) in retinal pigment epithelium. Biochemical and Biophysical Research Communications, v. 234, n. 1, p. 90-94, 8 maio 1997.

ZAJAC, A.; WAŚKIEWICZ, Z.; PILIS, W. Anaerobic power, creatine kinase activity, lactate concentration, and acid-base equilibrium changes following bouts of exhaustive strength exercises. Journal of strength and conditioning research / National Strength \& Conditioning Association, v. 15, n. 3, p. 357-361, ago. 2001. 